\title{
QUADRIC REPRESENTATION OF A SUBMANIFOLD
}

\author{
IVKO DIMITRIĆ
}

(Communicated by Jonathan M. Rosenberg)

\begin{abstract}
If $x: M^{n} \rightarrow E^{m}$ is an isometric immersion of a smooth manifold into a Euclidean space then the map $\tilde{x}=x x^{\mathfrak{t}}$ (t denotes transpose) is called the quadric representation of $M . \tilde{x}$ is said to be of finite type ( $k$-type) if it can be decomposed into a sum of finitely many $(k)$ eigenfunctions of Laplacian from different eigenspaces. We study map $\tilde{x}$ in general, especially as related to the condition of being of finite type. Certain classification results are obtained for manifolds with 1- and 2-type quadric representation.
\end{abstract}

\section{INTRODUCTION AND NOTATION}

Let $x: S^{m-1} \rightarrow E^{m}$ be the unit hypersphere centered at the origin embedded in the standard way. Regarding $x$ as a column matrix in $E^{m}$, the map $\tilde{x}=$ $x \cdot x^{\mathrm{t}}$ defines an isometric immersion of $S^{m-1}$ into the set $S M(m)$ of $m \times$ $m$ real symmetric matrices (this space becomes the standard Euclidean space of dimension $N=m(m+1) / 2$ when equipped with the metric $\tilde{g}(P, Q)=$ $\left.\frac{1}{2} \operatorname{tr}(P Q)\right)$, and this immersion is in fact the second standard immersion of sphere. It is well known that $\tilde{x}$ immerses $S^{m-1}$ as a minimal submanifold of a hypersphere of $S M(m)$ centered at $I / m$, where $I$ is $m \times m$ identity matrix, with radius $r=\sqrt{(m-1) / 2 m}$ (Veronese submanifold) (see e.g. [C2, $\mathrm{R}, \mathrm{S}]$ ). Obviously, $\tilde{x}$ also gives an isometric immersion of any submanifold $M^{n} \subset S^{m-1}$ (such submanifolds are called spherical). However, we can define this map more generally, that is, for an arbitrary submanifold $x: M^{n} \rightarrow E^{m}$, we put $\tilde{x}=x x^{\mathrm{t}}$ thus defining a smooth map $\tilde{x}: M^{n} \rightarrow S M(m)$ (in general not an isometric immersion). Since coordinates of $\tilde{x}$ depend on coordinates of $x$ in a quadratic manner, we call $\tilde{x}$ the quadric representation of submanifold $M$ and propose to study it.

A smooth map $f: M^{n} \rightarrow E^{m}$ is said to be of finite type ( $k$-type) if it can be decomposed into a sum of finitely many ( $k$, not counting a constant function) eigenfunctions of Laplacian of $M$ from different eigenspaces. In particular, a manifold isometrically immersed into a Euclidean space is of $k$-type if the corresponding immersion is of $k$-type. Thus $x: M \rightarrow E^{m}$ is $k$-type map if we can write (globally)

$$
x=x_{0}+x_{1}+\cdots+x_{k},
$$

Received by the editors December 5, 1989.

1980 Mathematics Subject Classification (1985 Revision). Primary 53C40, 53C42. 
where $x_{0}$ is a constant vector, and $\Delta x_{i}=\lambda_{i} x_{i}(i=1, \ldots, k)$, where $\lambda_{1}, \ldots, \lambda_{k}$ are $k$ different eigenvalues of Laplacian of $M$, and $\Delta$ acts on a vector function componentwise. In that case we have $P(\Delta)\left(x-x_{0}\right)=0$, where $P(T)=$ $\prod_{i=1}^{k}\left(T-\lambda_{i}\right)$. If $x$ cannot be represented as a finite sum (1), it is said to be an infinite type map. The notions of finite type maps and submanifolds was first introduced by B. Y. Chen and since then, many "nice" examples of submanifolds were shown to be of finite type (cf. [C2]). For example, a well-known result of Takahashi [T] asserts that the 1-type submanifolds are precisely those that are minimal in a hypersphere or minimal in the ambient Euclidean space.

In this paper we establish some general results about the quadric representation, in particular those related to the condition of $\tilde{x}$ being of finite type. Studying submanifolds whose quadric representation is of finite type amounts to studying spectral behavior of products of coordinate functions $x_{i} \cdot x_{j}$. We classify submanifolds for which $\tilde{x}$ is a 1-type map as totally geodesic spherical submanifolds. Although construction of nonspherical submanifolds for which $\tilde{x}$ is finite type is relatively easy, we show that minimal submanifolds of $E^{m}$ have quadric representation of infinite type. Also, a result on hypersurfaces with 2-type quadric representation is proved.

Let us fix the notation (standard facts from submanifold theory can be found in [C1]). Let $M^{n}$ be a submanifold of the Euclidean space $E^{m}$. Suppose that $e_{1}, e_{2}, \ldots, e_{n}, e_{n+1}, \ldots, e_{m}$ are local orthonormal vector fields along $M$ such that the first $n$ vectors are tangent to $M$ and the remaining $m-n$ vectors normal to $M$. Let $\langle$,$\rangle and \bar{\nabla}$ be the Euclidean metric and connection of $E^{m}$, and denote by $\nabla, h, D, A_{\xi}$ respectively, the induced connection, second fundamental form of $M$, connection in the normal bundle $T^{\perp} M$, and the Weingarten endomorphism relative to the normal direction $\xi$. They are defined by the following equations:

$$
\bar{\nabla}_{X} Y=\nabla_{X} Y+h(X, Y), \quad \bar{\nabla}_{X} \xi=-A_{\xi} X+D_{X} \xi
$$

for $X, Y \in T M$ and $\xi \in T^{\perp} M$, where first terms on the right-hand sides of these equations are tangent to $M$ and the second terms are normal to $M$. Instead of $A_{e_{r}}$ we write $A_{r}$ for short. The connection forms $\omega_{i}^{j}$ and the mean curvature vector $H$ of $M$ in $E^{m}$ are defined by $\nabla_{e_{k}} e_{i}=\sum_{j} \omega_{i}^{j}\left(e_{k}\right) e_{j}$, $H=(1 / n) \sum_{r}\left(\operatorname{tr} A_{r}\right) e_{r}$. In this setting indices $i, j, k$ always range from 1 to $n$ and indices $r, s$ from $n+1$ to $m$. If we choose $e_{n+1} \| H$ then $H=\alpha e_{n+1}$ for some function $\alpha$ called the mean curvature. As usual, $\Delta$ denotes Laplacian of $M$ acting on smooth functions, i.e. $\Delta f=\sum_{i=1}^{n}\left[\left(\nabla_{e_{i}} e_{i}\right) f-e_{i}\left(e_{i} f\right)\right]$. The metric on $S M(m)$ is given by $\tilde{g}(P, Q)=\frac{1}{2} \operatorname{tr}(P Q)$ and the Euclidean connection there is denoted by $\widetilde{\nabla}$. We define a map $*$ from $E^{m} \times E^{m}$ into $S M(m)$ by $X * Y=X Y^{\mathrm{t}}+Y X^{\mathrm{t}}$ for column vectors $X, Y$ in $E^{m}$. Then $X * Y=Y * X$ and $*$ is bilinear, $\widetilde{\nabla}_{X}(Y * Z)=\left(\bar{\nabla}_{X} Y\right) * Z+Y *\left(\bar{\nabla}_{X} Z\right)$ and $\tilde{g}(X * Y, Z * W)=$ $\langle X, W\rangle\langle Y, Z\rangle+\langle Y, W\rangle\langle X, Z\rangle$.

We assume all manifolds smooth and connected and all immersions smooth.

\section{QUADRIC REPRESENTATION OF A SUBMANIFOLD}

First we prove a theorem about quadric representation being an isometric immersion. 
Theorem 1. Let $x: M^{n} \rightarrow E^{m}$ be an isometric immersion of a Riemannian manifold into a Euclidean space. Then

(i) $\tilde{x}=x x^{t}$ is an isometric immersion if and only if $x\left(M^{n}\right) \subset S^{m-1}$, i.e. $M$ is spherical. (In the case $n=1$, a curve is assumed to be complete.) Moreover,

(ii) If $n>1, \tilde{x}$ is a conformal map if and only if $M^{n} \subset S^{m-1}(r)$, in which case $\tilde{x}$ is homothety.

Proof. (i) First we prove the statement for a complete curve $x: C \rightarrow E^{m}$. Let $x(s)=\left(x_{1}(s), x_{2}(s), \ldots, x_{m}(s)\right)$ be the parametrization of the curve by its arclength. Then

$$
d \tilde{x} \otimes d \tilde{x}=\frac{1}{2} \operatorname{tr}(d \tilde{x} d \tilde{x})=\frac{1}{2} \sum_{i, j}\left(x_{i}^{\prime} x_{j}+x_{i} x_{j}^{\prime}\right)^{2} d s^{2} .
$$

Since $\tilde{x}$ is assumed to be an isometry, tangent vector $d \tilde{x} / d s$ must have length 1 ; therefore, we get

$$
\begin{aligned}
1 & =\frac{1}{2} \sum_{i, j}\left(x_{i}^{\prime} x_{j}+x_{i} x_{j}^{\prime}\right)^{2}=2\left(\sum_{i} x_{i} x_{i}^{\prime}\right)^{2}+\sum_{i<j}\left(x_{i}^{\prime} x_{j}-x_{i} x_{j}^{\prime}\right)^{2} \\
& =\frac{1}{2}\left[\left(\|x\|^{2}\right)^{\prime}\right]^{2}+\left\|x \wedge x^{\prime}\right\|^{2}=\frac{1}{2}\left[\left(\|x\|^{2}\right)^{\prime}\right]^{2}+\|x\|^{2}-\frac{1}{4}\left[\left(\|x\|^{2}\right)^{\prime}\right]^{2} \\
& =\|x\|^{2}+\frac{1}{4}\left[\left(\|x\|^{2}\right)^{\prime}\right]^{2} .
\end{aligned}
$$

Thus letting $u(s)=\|x\|^{2}$, we get the differential equation in $u$ that separates the variables, $u+(1 / 4)\left(u^{\prime}\right)^{2}=1$. One obvious solution is $u=1$, and there is no solution $u>1$. If $u \neq 1$, solving the equation we get $u(s)=1-$ $(c+s)^{2}$, where $c$ is an arbitrary constant. This solution, however, represents a decreasing function of $s$ and therefore, $u=\|x\|^{2}<0$ for sufficiently large $s$ (curve is assumed to be complete), which is a contradiction. Therefore, $u=1$ i.e. curve $C$ belongs to the unit sphere centered at the origin. If a curve is not assumed complete, then there are nonspherical examples for which $\tilde{x}$ is an isometry e.g. curve in $E^{2}$ given by $x(s)=\sqrt{1-s^{2}} \cos \theta(s), y(s)=\sqrt{1-s^{2}} \sin \theta(s)$, with $\theta^{\prime}(s)=\sqrt{1-2 s^{2}} /\left(1-s^{2}\right), 0<s<\sqrt{2} / 2$.

Now suppose $\tilde{x}$ is an isometric immersion for a manifold $M^{n}(n>1)$. Let $p \in M$ be an arbitrary point, and consider a small smooth loop based at $p$. The restriction of $\tilde{x}$ to this loop is an isometry, and from the above we conclude that the loop belongs to the unit sphere centered at the origin and the same is true for point $p$. Since $p$ is an arbitrary point, $M$ is a spherical submanifold. The converse of the statement is well known.

(ii) Since $\tilde{\nabla}$ acts as a derivation on the set of smooth functions on $M$, we have

$$
\widetilde{\nabla}_{X} \tilde{x}=\widetilde{\nabla}_{X}\left(x x^{\mathfrak{t}}\right)=\left(\bar{\nabla}_{X} x\right) x^{\mathrm{t}}+x\left(\bar{\nabla}_{X} x\right)^{\mathrm{t}}=X x^{\mathrm{t}}+x X^{\mathrm{t}}=X * x .
$$

If $\tilde{x}$ is a conformal map it maps a pair of perpendicular vectors into a pair of perpendicular vectors. Therefore, if $X \perp Y$ we have

$$
\begin{aligned}
0 & =\tilde{g}(d \tilde{x}(X), d \tilde{x}(Y))=\tilde{g}\left(\widetilde{\nabla}_{X} \tilde{x}, \tilde{\nabla}_{Y} \tilde{x}\right) \\
& =\tilde{g}(X * x, Y * x)=\langle x, X\rangle\langle x, Y\rangle .
\end{aligned}
$$

If $X, Y$ is a pair of tangent perpendicular unit vectors then $X+Y$ and $X-Y$ are also perpendicular, and from the above equation we obtain

$$
0=\langle x, X+Y\rangle\langle x, X-Y\rangle=\langle x, X\rangle^{2}-\langle x, Y\rangle^{2} .
$$


Therefore, $\langle x, X\rangle=0$ for every tangent vector $X$ of $M$, and hence $\langle x, x\rangle=$ $r^{2}=$ const, which shows that $x(M) \subset S^{m-1}(r)$. The converse is clear because then $g\left(\tilde{x}_{*} X, \tilde{x}_{*} Y\right)=\langle x, x\rangle\langle X, Y\rangle$.

If $x: M^{n} \rightarrow E^{m}$ is a spherical submanifold, i.e. submanifold of the unit hypersphere centered at the origin, then $\tilde{x}$ is also an isometric immersion by virtue of $\tilde{x}_{*}(X)=X x^{\mathrm{t}}+x X^{\mathrm{t}}$. It is interesting to see how certain properties of the immersion $x$ are reflected in the immersion $\tilde{x}$ and vice versa. To that end we prove the following theorem. We use symbols with $\sim$ for objects related to the immersion $\tilde{x}$, those without $\sim$ are related to the immersion into $E^{m}$ and symbols with ' relate to the immersion into $S^{m-1}$.

Theorem 2. Let $x: M^{n} \rightarrow S^{m-1} \subset E^{m}$ be an isometric immersion, and let $\tilde{x}: M^{n} \rightarrow S M(m)$ be its quadric representation. Then

(i) $\|\tilde{h}\|=$ const $\Leftrightarrow\|h\|=$ const,$\|\tilde{H}\|=$ const $\Leftrightarrow\|H\|=$ const .

(ii) $M^{n}$ is pseudoumbilical in $S M(m)$ via $\tilde{x} \Leftrightarrow M^{n}$ is pseudoumbilical in $E^{m}$ via $x$. (A submanifold is called pseudoumbilical if $A_{H}=\mu I$ for some function $\mu$.)

(iii) $\widetilde{D} \widetilde{H}=0 \Leftrightarrow h^{\prime}=0$, i.e. $M^{n}$ is totally geodesic in $S^{m-1}$.

(iv) $\tilde{\nabla} \tilde{h}=0 \Leftrightarrow h^{\prime}=0$.

Proof. Since $\tilde{x}$ is an isometric immersion, we have $\Delta \tilde{x}=-n \widetilde{H}$. On the other hand by the product formula for Laplacian,

$$
\Delta \tilde{x}=\frac{1}{2} \Delta(x * x)=(\Delta x) * x-\sum_{i}\left(\bar{\nabla}_{e_{i}} x\right) *\left(\bar{\nabla}_{e_{i}} x\right)=-n H * x-\sum_{i} e_{i} * e_{i} .
$$

Therefore,

$$
\widetilde{H}=H * x+\frac{1}{n} \sum_{i} e_{i} * e_{i} .
$$

Using the fact that $H=H^{\prime}-x$, we obtain

$$
\|\widetilde{H}\|^{2}=\frac{1}{2} \operatorname{tr}\left(\widetilde{H}^{2}\right)=\left\|H^{\prime}\right\|^{2}+2+\frac{2}{n}=\|H\|^{2}+\frac{2+n}{n} .
$$

This proves second equivalence of (i) (cf. [C2, Lemma 4.6.4, p. 152]). The first equivalence can be proved using similar reasoning. In fact

$$
\tilde{h}(X, Y)=\tilde{x}_{*}\left(h^{\prime}(X, Y)\right)+X * Y-\langle X, Y\rangle x * x,
$$

and hence $\|\tilde{h}\|^{2}=\|h\|^{2}+n^{2}+2 n$. To prove (ii) and (iii) we differentiate (3) along a vector field $X \in T M$ to get

$$
\begin{aligned}
\widetilde{\nabla}_{X} \widetilde{H} & =\widetilde{\nabla}_{X}(H * x)+\frac{1}{n} \sum_{i} \widetilde{\nabla}_{X}\left(e_{i} * e_{i}\right) \\
& =-\left(A_{H} X\right) * x+\left(D_{X} H\right) * x+H * X+\frac{2}{n} \sum_{r=n+1}^{m} e_{r} *\left(A_{r} X\right) .
\end{aligned}
$$

We simplify this by choosing $e_{m}=x$, and observing that $H=H^{\prime}-x$, we 
obtain

$$
\begin{aligned}
\tilde{\nabla}_{X} \widetilde{H}= & -\left[\tilde{x}_{*}\left(A_{H} X\right)+\frac{n+2}{n} \tilde{x}_{*}(X)\right] \\
& +\left(D_{X} H^{\prime}\right) * x+H^{\prime} * x+\frac{2}{n} \sum_{r=n+1}^{m-1} e_{r} *\left(A_{r} X\right) .
\end{aligned}
$$

On the other hand, $\widetilde{\nabla}_{X} \widetilde{H}=-\widetilde{A}_{\widetilde{H}} X+\widetilde{D}_{X} \widetilde{H}$; so by comparing components of (4) that are tangent and normal to $M$, we obtain

$$
\widetilde{A}_{\widetilde{H}} X=A_{H} X+\frac{n+2}{n} X
$$

$$
\widetilde{D}_{X} \widetilde{H}=\left(D_{X} H^{\prime}\right) * x+H^{\prime} * X+\frac{2}{n} \sum_{r=n+1}^{m-1} e_{r} *\left(A_{r} X\right) .
$$

Equation (5) proves (ii). Note that on the right-hand side of (6), $\left(D_{X} H^{\prime}\right) * x$ is the component tangent to the sphere $S^{m-1}$ via $\tilde{x}$, and the rest represents component of $\widetilde{D}_{X} \widetilde{H}$, which is normal to $S^{m-1}$ because $T_{P}^{\perp} S^{m-1}=\{\xi \in$ $S M(m) \mid P \xi=\xi P\} \quad$ (see [R]). Therefore, if $\widetilde{D}_{X} \widetilde{H}=0$, we see that $D^{\prime} H^{\prime}=0$ and

$$
H^{\prime} * X+\frac{2}{n} \sum_{r=n+1}^{m-1} e_{r} *\left(A_{r} X\right)=0
$$

Using $H^{\prime}=\frac{1}{n} \sum_{r=n+1}^{m-1}\left(\operatorname{tr} A_{r}\right) e_{r}$ and substituting into (7) we obtain

$$
\frac{1}{n} \sum_{r=n+1}^{m-1} e_{r} *\left[\left(\operatorname{tr} A_{r}\right) X+2\left(A_{r} X\right)\right]=0, \quad \text { for every } X \in T M .
$$

From here we have $\left(\operatorname{tr} A_{r}\right) I+2 A_{r}=0$, and taking trace of this relation, we get $(n+2) \operatorname{tr} A_{r}=0$, i.e. $\operatorname{tr} A_{r}=0$. Putting this back into the relation we conclude $A_{r}=0$ for every $r=n+1, \ldots, m-1$, equivalently $h^{\prime}=0$, which means that $M^{n}$ is totally geodesic in $S^{m-1}$. Conversely, if $h^{\prime}=0$, then $\tilde{x}$ immerses $M$ as a minimal submanifold of a hypersphere of $S M(m)$ centered at $I_{n+1} /(n+1)$ in $S M(m)$ (Veronese submanifold) so that $\widetilde{H}$ is a constant multiple of $\left(\tilde{x}-I_{n+1} / n+1\right)$, and therefore $\widetilde{D} \widetilde{H}=0$ proving (iii).

Part (iv) follows from (iii) because $\tilde{\nabla} \tilde{h}=0$ implies $\widetilde{D} \widetilde{H}=0$.

\section{Submanifolds With FINITE TYPE $\tilde{x}$}

Since a 1-type map is next simplest to being harmonic, we start out by proving a theorem about submanifolds with 1-type $\tilde{x}$.

Theorem 3. For an isometric immersion $x: M^{n} \rightarrow E^{m}, \tilde{x}$ is 1-type if and only if $M^{n}$ is a totally geodesic submanifold of the hypersphere $S^{m-1}(r) \subset E^{m}$ centered at the origin. In particular, if the immersion $x$ is full and $M$ complete, then $M=S^{m-1}(r)$ is the standard sphere.

Proof. Suppose that $\tilde{x}$ is a 1-type map. Then we can write $\tilde{x}=\tilde{x}_{0}+\tilde{x}_{p}$, where $\tilde{x}_{0}$ is a constant vector and $\Delta \tilde{x}_{p}=\lambda_{p} \tilde{x}_{p}$, i.e. $\tilde{x}_{p}$ is an eigenvector of the 
Laplacian. Thus,

$$
\Delta \tilde{x}=\lambda_{p} \tilde{x}_{p}=\lambda_{p}\left(\tilde{x}-\tilde{x}_{0}\right) .
$$

Therefore, from (2) and (8) we have $-n H * x-\sum_{i} e_{i} * e_{i}=\lambda_{p}\left(\tilde{x}-\tilde{x}_{0}\right)$. Differentiating this relation along an arbitrary vector field $X$ of $M$, we get

$$
\begin{aligned}
\lambda_{p} X * x= & n\left(A_{H} X\right) * x-n\left(D_{X} H\right) * x-n H * X \\
& -2 \sum_{i, j} \omega_{i}^{j}(X)\left(e_{i} * e_{j}\right)-2 \sum_{i} h\left(X, e_{i}\right) * e_{i} .
\end{aligned}
$$

Note that the first sum is equal to 0 , since $\omega_{i}^{j}$ is antisymmetric in $i$ and $j$ whereas $e_{i} * e_{j}$ is symmetric in $i, j$. Also

$$
\sum_{i} h\left(X, e_{i}\right) * e_{i}=\sum_{i, r}\left\langle h\left(X, e_{i}\right), e_{r}\right\rangle\left(e_{i} * e_{r}\right)=\sum_{r}\left(A_{r} X\right) * e_{r},
$$

and therefore, for every $X \in T M$ we have

$$
n\left(A_{H} X\right) * x-n\left(D_{X} H\right) * x-n H * X-2 \sum_{r}\left(A_{r} X\right) * e_{r}=\lambda_{p} X * x
$$

We now find $e_{i} * e_{r}$ component of (9), i.e. apply $\tilde{g}\left(-, e_{i} * e_{r}\right)$ to it:

$$
\begin{aligned}
& n\left\langle A_{H} X, e_{i}\right\rangle\left\langle x, e_{r}\right\rangle-n\left\langle D_{X} H, e_{r}\right\rangle\left\langle x, e_{i}\right\rangle-n\left\langle H, e_{r}\right\rangle\left\langle X, e_{i}\right\rangle-2\left\langle A_{r} X, e_{i}\right\rangle \\
& \quad=\lambda_{p}\left\langle X, e_{i}\right\rangle\left\langle x, e_{r}\right\rangle .
\end{aligned}
$$

Letting $X=e_{i}$ and summing on $i$, we get

$$
n\left(\operatorname{tr} A_{H}\right)\left\langle x, e_{r}\right\rangle-n\left\langle D_{x_{T}} H, e_{r}\right\rangle-n^{2}\left\langle H, e_{r}\right\rangle-2 \operatorname{tr} A_{r}=n \lambda_{p}\left\langle x, e_{r}\right\rangle .
$$

If we multiply this relation by $e_{r}$ and sum on $r$, we obtain

$$
\left(\operatorname{tr} A_{H}-\lambda_{p}\right) x_{N}-D_{x_{T}} H=(n+2) H .
$$

Note that in general we have both normal and tangential component of $x$ :

$$
x_{N}=\sum_{r}\left\langle x, e_{r}\right\rangle e_{r}, \quad x_{T}=\sum_{i}\left\langle x, e_{i}\right\rangle e_{i} .
$$

Finding $e_{r} * e_{r}$ component of (9) and summing on $r$, we get

$$
\left\langle D_{X} H, x_{N}\right\rangle=0 \text { i.e. } D_{X} H \perp x_{N} \text { for every } X \in T M \text {. }
$$

Finding $e_{r} * e_{s}$ component of (9) and summing on $s$ (after multiplying by $e_{s}$ ), we obtain

$\left\langle D_{X} H, e_{r}\right\rangle x_{N}=\left\langle x, e_{r}\right\rangle D_{X} H=0, \quad$ for every $r=n+1, \ldots, m$ and $X \in T M$.

Thus, at any given point of $M$ we have

$$
x_{N}=0 \text { or } x_{N} \neq 0 \text { and } D H=0 .
$$

Next, by comparing $e_{k} * e_{k}$ components of two sides of the equation (9), multiplying by $e_{k}$ and summing on $k$, we get

$$
\left\langle n A_{H} X-\lambda_{p} X, x_{T}\right\rangle=0, \quad \text { for every } X \in T M,
$$

and by comparing $e_{i} * e_{k}$ components, summing on $k$ and using (12),

$$
\left[n\left\langle A_{H} X, e_{i}\right\rangle-\lambda_{p}\left\langle X, e_{i}\right\rangle\right] x_{T}=\left(n A_{H} X-\lambda_{p} X\right)\left\langle x, e_{i}\right\rangle=0,
$$


for every $i=1,2, \ldots, n$ and every $X \in T M$. Therefore, at any given point of $M$ we have

$$
x_{T}=0 \text { or } x_{T} \neq 0 \text { and } n A_{H}=\lambda_{p} I .
$$

Let $U=\left\{p \in M \mid x_{T} \neq 0\right.$ at $\left.p\right\}$. Then $U$ is an open subset of $M$, and on $U$ we have by (13), $\operatorname{tr} A_{H}=\lambda_{p}$. Then (10) implies $D_{x_{T}} H=-(n+2) H$ on $U$. Now let $V$ be an open subset of $U$ defined by $V=\left\{p \in U \mid x_{N} \neq 0\right.$ at $\left.p\right\}$. By (11) we have $D H=0$ on $V$, and from the above we conclude $H=0$ on $V$, i.e. $V$ is the piece of $M$ immersed minimally in $E^{m}$. Now we compute $\operatorname{tr}(\Delta \tilde{x})$ on $V$, noting that the Laplacian commutes with trace since it is a linear operator.

$$
\begin{aligned}
\operatorname{tr}(\Delta \tilde{x}) & =\Delta(\operatorname{tr} \tilde{x})=\Delta\langle x, x\rangle=2\langle\Delta x, x\rangle-2 \sum_{i}\left\langle e_{i}, e_{i}\right\rangle \\
& =-2 n\langle H, x\rangle-2 n=-2 n .
\end{aligned}
$$

On the other hand, $(8)$ yields

$$
\operatorname{tr}(\Delta \tilde{x})=\lambda_{p}\left(\operatorname{tr} \tilde{x}-\operatorname{tr} \tilde{x}_{0}\right)=\lambda_{p}\left(\langle x, x\rangle-\operatorname{tr} \tilde{x}_{0}\right) .
$$

Therefore, $\lambda_{p}\left(\langle x, x\rangle-\operatorname{tr} \tilde{x}_{0}\right)=-2 n$, and since obviously $\lambda_{p} \neq 0$ we have

$$
\langle x, x\rangle=\operatorname{tr} \tilde{x}_{0}-2 n / \lambda_{p}=\text { const } .
$$

Consequently, $x(V) \subset S^{m-1}(r)$ and hence $0=H=H^{\prime}-x / r^{2}$, where $H^{\prime}$ is the mean curvature vector of $V$ in $S^{m-1}(r)$, which is a contradiction because $H^{\prime} \perp x$ and $x \neq 0$. Therefore, we must have $V=\varnothing$, and hence on $U x=x_{T}$ is tangential. Now on $U$ as before we have (note $H \perp x) \operatorname{tr}(\Delta \tilde{x})=-2 n=$ $\lambda_{p}\left(\langle x, x\rangle-\operatorname{tr} \tilde{x}_{0}\right)$, and therefore (14) holds again on $U$. So, $x(U) \subset S^{m-1}(r)$ but then $x_{T}=0$, which is a contradiction. We conclude $U=\varnothing$, and $x=x_{N}$ is normal to submanifold $M$. Consequently, $x$ immerses $M$ into a hypersphere of $E^{m}$ centered at the origin $x: M^{n} \rightarrow S^{m-1}(r) \subset E^{m}$. In that case $H=H^{\prime}-$ $x / r^{2}$. From (11) we get $D H=0$, and then from (10) it follows $\left(\operatorname{tr} A_{H}-\lambda_{p}\right) x=$ $(n+2)\left(H^{\prime}-x / r^{2}\right)$. Since $H^{\prime} \perp x$ we see that $H^{\prime}=0$ i.e. $M$ is minimal in the hypersphere. With these identities in effect, equation (9) becomes (we take $\left.e_{m}=x / r\right)$ :

$$
\frac{2(n+1)}{r^{2}} X * x-2 \sum_{r=1}^{m-1}\left(A_{r} X\right) * e_{r}=\lambda_{p} X * x,
$$

for every $X \in T M$. Therefore, $\lambda_{p}=2(n+1) / r^{2}$ and $A_{s}=0$ for every $s=n+1, \ldots, m-1$. We conclude that $M^{n}$ is totally geodesic in $S^{m-1}(r)$, i.e. it is (a piece of) the standard $S^{n}(r)$ in $S^{m-1}(r)$.

Conversely, if $M^{n}$ is the totally geodesic $S^{n}(r) \subset S^{m-1}(r)$ then it is well known that $M^{n}$ is minimally immersed via $\tilde{x}$ (after scaling the metric in $S M(m)$ with factor $\left.1 / r^{2}\right)$ as a Veronese submanifold in a hypersphere of $S M(m)$. Then the well-known theorem of Takahashi [T, C2] asserts that $\tilde{x}$ is of 1-type. As a matter of fact we have

$$
\tilde{x}=\tilde{x}_{0}+\tilde{x}_{p}=\frac{1}{n+1}\left(x x^{\mathrm{t}}+r^{2} \sum_{i} e_{i} e_{i}^{\mathrm{t}}\right)+\frac{1}{n+1}\left(n x x^{\mathrm{t}}-r^{2} \sum_{i} e_{i} e_{i}^{\mathrm{t}}\right),
$$


where $\left(x x^{\mathrm{t}}+r^{2} \sum_{i} e_{i} e_{i}^{\mathrm{t}}\right)$ is a constant vector, actually equal to $r^{2} I_{n+1}$ in $S M(m)$, and $\left(n x x^{\mathrm{t}}-r^{2} \sum_{i} e_{i} e_{i}^{\mathrm{t}}\right)$ is an eigenvector of the Laplacian corresponding to the second nonzero eigenvalue $\lambda_{p}=2(n+1) / r^{2}$ of $S^{n}(r)$ (cf. [R]).

It is known that a closed curve in $E^{m}$ is of finite type if and only if Fourier series expansions of its coordinate functions have finitely many nonzero terms (see e.g. [C2]). There are nonspherical closed curves in $E^{m}$ of finite type (see [C2, p. 289]). They are also of finite type ( $\tilde{x}$ not an isometric immersion) in $S M(m)$ via $\tilde{x}$ since by the product formulas of trigonometry their Fourier series expansions still have finitely many nonzero terms. We also have the following example:

Example. Given the two nonspherical finite type curves $C_{1}, C_{2}$ mentioned above, consider their product $C_{1} \times C_{2}$. Such product does not belong to any sphere and its quadric representation is of finite type since the Laplacian of a product splits into the sum of Laplacians on the component manifolds.

However, we are able to prove the following theorem for minimal submanifolds.

Theorem 4. For a minimal immersion $x: M^{n} \rightarrow E^{m}$, the quadric representation $\tilde{x}$ is of infinite type.

Proof. Suppose $\tilde{x}$ is a $k$-type map where $k$ is finite. Then we can decompose $\tilde{x}$ as $\tilde{x}=\tilde{x}_{0}+\tilde{x}_{1}+\tilde{x}_{2}+\cdots+\tilde{x}_{k}$, where $\tilde{x}_{0}=$ const and $\Delta \tilde{x}_{i}=\lambda_{i} \tilde{x}_{i}$, $i=1, \ldots, k$. Finding successively iterated Laplacians $\Delta \tilde{x}, \ldots, \Delta^{k} \tilde{x}$ and eliminating $\tilde{x}_{1}, \tilde{x}_{2}, \ldots, \tilde{x}_{k}$ from these $k+1$ equations, we obtain

$$
\sigma_{k}\left(\tilde{x}-\tilde{x}_{0}\right)+\sigma_{k-1} \Delta\left(\tilde{x}-\tilde{x}_{0}\right)+\cdots+\sigma_{1} \Delta^{k-1}\left(\tilde{x}-\tilde{x}_{0}\right)+\Delta^{k}\left(\tilde{x}-\tilde{x}_{0}\right)=0,
$$

where $\sigma_{i}$ is the $i$ th elementary symmetric function of $\lambda_{1}, \lambda_{2}, \ldots, \lambda_{k}$ that is $\sigma_{1}=-\left(\lambda_{1}+\cdots+\lambda_{k}\right), \ldots, \sigma_{k}=(-1)^{k} \lambda_{1} \cdots \lambda_{k}$. As before,

$$
\operatorname{tr}(\Delta \tilde{x})=\Delta\langle x, x\rangle=2\langle\Delta x, x\rangle-2 \sum_{i}\left\langle e_{i}, e_{i}\right\rangle=-2 n
$$

and by iterating we get $\operatorname{tr}\left(\Delta^{i} \tilde{x}\right)=0$ for $i \geq 2$. Hence by taking trace of (15), we obtain

$$
\sigma_{k}\left(\operatorname{tr} \tilde{x}-\operatorname{tr} \tilde{x}_{0}\right)-2 n \sigma_{k-1}=0 .
$$

$1^{\circ}$. If $\sigma_{k} \neq 0$ then $\langle x, x\rangle=\operatorname{tr} \tilde{x}=\operatorname{tr} \tilde{x}_{0}+2 n \sigma_{k-1} / \sigma_{k}=$ const, so $x\left(M^{n}\right) \subset$ $S^{m-1}(r)$. But a spherical submanifold cannot be minimal in ambient Euclidean space, therefore we have a contradiction.

$2^{\circ}$. If $\sigma_{k}=0$, then one eigenvalue, say $\lambda_{1}$, must be zero. If $k \geq 2$ we conclude from (16) that also $\sigma_{k-1}=0$. That implies that another eigenvalue, say $\lambda_{2}$, is zero, which is a contradiction because $\lambda_{1}$ and $\lambda_{2}$ are different eigenvalues. If $k=1$ (and $\left.\sigma_{1}=0\right)$ then $\tilde{x}=\tilde{x}_{0}+\tilde{x}_{1}$ with $\Delta \tilde{x}=0$, so by taking trace, $0=\operatorname{tr}(\Delta \tilde{x})=-2 n$, which again is a contradiction. We conclude that $\tilde{x}$ cannot be a finite type map.

Studying submanifolds with quadric representation $\tilde{x}$ of type $\geq 2$ is difficult in general, even when a submanifold is assumed to have constant mean curvature $\alpha$ in $E^{m}$. However, we have the following result for hypersurfaces. 
Theorem 5. The only constant mean curvature hypersurface $x: M^{n} \rightarrow E^{n+1}$ with 2-type quadric representation $\tilde{x}$ is (a piece of) a hypersphere which is not centered at the origin.

Proof. Let $\xi$ be a local orthonormal field of unit normals to $M$ so that $H=\alpha \xi$. We compute $\Delta \xi=\|A\|^{2} \xi$, where $\|A\|^{2}=\operatorname{tr} A^{2}$ is the square of the norm of the second fundamental form. Starting from (2), $\Delta \tilde{x}=-n \alpha \xi * x-\sum_{i} e_{i} * e_{i}$, we compute $\Delta^{2} \tilde{x}$ using the product formula for the Laplacian:

$$
\Delta(X * Y)=(\Delta X) * Y+X *(\Delta Y)-2 \sum_{i}\left(\bar{\nabla}_{e_{i}} X\right) *\left(\bar{\nabla}_{e_{i}} Y\right) .
$$

We get

(17)

$\Delta^{2} \tilde{x}=-n \alpha\|A\|^{2} \xi * x+\left(n^{2} \alpha^{2}+2\|A\|^{2}\right) \xi * \xi-2 n \alpha \sum_{i}\left(A e_{i}\right) * e_{i}-2 \sum_{i}\left(A e_{i}\right) *\left(A e_{i}\right)$.

Suppose that $\tilde{x}$ is a 2-type map so that $\tilde{x}=\tilde{x}_{0}+\tilde{x}_{1}+\tilde{x}_{2}$ where $\tilde{x}_{0}=$ const, $\Delta \tilde{x}_{i}=\lambda_{i} \tilde{x}_{i}, i=1,2$. Then

$$
\Delta^{2} \tilde{x}-\left(\lambda_{1}+\lambda_{2}\right) \Delta \tilde{x}+\lambda_{1} \lambda_{2}\left(\tilde{x}-\tilde{x}_{0}\right)=0 .
$$

Denote the left-hand side of $(18)$ by $Q(\tilde{x})$, and let $X$ be any vector field tangent to $M$. Then using (2), (17), and (18), we obtain

$$
\begin{aligned}
0=\tilde{g}\left(\tilde{\nabla}_{X} Q(\tilde{x}), \xi * \xi\right) & =X \tilde{g}(Q(\tilde{x}), \xi * \xi)+2 \tilde{g}(Q(\tilde{x}),(A X) * \xi) \\
& =4\left\langle X, \nabla\|A\|^{2}\right\rangle-2 n \alpha\left\langle X, \nabla\|A\|^{2}\right\rangle\langle x, \xi\rangle .
\end{aligned}
$$

Define a closed set $S=\left\{p \in M \mid \nabla\|A\|^{2}=0\right.$ at $\left.p\right\}$. Then on the open set $U=$ $M \backslash S$ we have $\langle x, H\rangle=2 / n=$ const. Since $\Delta x=-n H$, this implies $\langle\Delta x, x\rangle=-2$ and hence $\operatorname{tr}(\Delta \tilde{x})=\Delta\langle x, x\rangle=2\langle\Delta x, x\rangle-2 n=-2(n+2)$, $\operatorname{tr}\left(\Delta^{2} \tilde{x}\right)=0$. Therefore, taking trace of $(18)$ yields $2(n+2)\left(\lambda_{1}+\lambda_{2}\right)+$ $\lambda_{1} \lambda_{2}\left(\langle x, x\rangle-\operatorname{tr} \tilde{x}_{0}\right)=0$ and consequently $\langle x, x\rangle=$ const. This means that $U \subset S^{n}(r)$ and by Theorem 3, $\tilde{x}$ would be a 1-type map, which is a contradiction (a constant mean curvature hypersurface is analytic). Thus $U$ is empty and $\|A\|^{2}=$ const on $M$. Moreover, we see that $x_{T}=0$ cannot hold on an open set of $M$. Next we compute $\operatorname{tr}\left(\Delta^{2} \tilde{x}\right)$ using (17) to get $\operatorname{tr}\left(\Delta^{2} \tilde{x}\right)=-2 n \alpha\left(\|A\|^{2}\langle x, \xi\rangle+n \alpha\right)$. Therefore, trace of (18) becomes

$-2 n \alpha\left(\|A\|^{2}\langle x, \xi\rangle+n \alpha\right)=2 n\left(\lambda_{1}+\lambda_{2}\right)(1+\alpha\langle x, \xi\rangle)+\lambda_{1} \lambda_{2}\left(\langle x, x\rangle-\operatorname{tr} \tilde{x}_{0}\right)=0$.

Differentiating this equation with respect to an arbitrary tangent vector field $X$ we get $n \alpha\left(\|A\|^{2}-\lambda_{1}-\lambda_{2}\right)\langle A X, x\rangle+\lambda_{1} \lambda_{2}\langle X, x\rangle=0$. If $\|A\|^{2}=\lambda_{1}+\lambda_{2}$ then $\lambda_{1}=0$ and the equation above gives $\|A\|^{2}=\lambda_{2}=n \alpha^{2}$. Then (18) becomes $\Delta^{2} \tilde{x}=n \alpha^{2} \Delta \tilde{x}$. However, this is easily seen to be impossible by (2) and (17). If $\|A\|^{2} \neq \lambda_{1}+\lambda_{2}$ then from the above we have $A x_{T}=\mu x_{T}$ with $\mu=\lambda_{1} \lambda_{2} / n \alpha\left(\lambda_{1}+\lambda_{2}-\|A\|^{2}\right)=$ const. Let $e_{1}, e_{2}, \ldots, e_{n}$ be principal directions of $M$ with $e_{1}$ in the direction of $x_{T}$ i.e. $x_{T}=\nu e_{1}$ and let $\mu_{1}, \ldots, \mu_{n}$ be the corresponding principal curvatures $\left(\mu_{1}=\mu\right)$. Since

$$
X=\bar{\nabla}_{X} x=\nabla_{X} x_{T}+h\left(X, x_{T}\right)-A_{x_{N}} X+D_{X} x_{N},
$$

by comparing parts tangent to $M$, we get

$$
\nabla_{X} x_{T}=X+A_{x_{N}} X \text {. }
$$


Substituting $X=x_{T}=\nu e_{1}$ into this equation, we get $\nabla_{e_{1}} e_{1}=0$ and $e_{1} \nu=1+$ $\mu\langle x, \xi\rangle$. Putting $X=e_{k}, k \geq 2$ into (19), we obtain $\nu \omega_{1}^{k}\left(e_{k}\right)=1+\mu_{k}\langle x, \xi\rangle$. From the Codazzi equation $\left(\nabla_{e_{1}} A\right) e_{k}=\left(\nabla_{e_{k}} A\right) e_{1}$ for $\mu_{k} \neq \mu$, by comparing $e_{k}$ components we have

$$
e_{1} \mu_{k}=\left(\mu-\mu_{k}\right) \omega_{1}^{k}\left(e_{k}\right)=\frac{1}{\nu}\left(\mu-\mu_{k}\right)\left(1+\mu_{k}\langle x, \xi\rangle\right) .
$$

Let $p=\#\left\{k \mid \mu_{k}=\mu\right\}$. Then from (20) by adding on $k$, we obtain $0=$ $e_{1}(n \alpha-p \mu)=\frac{1}{\nu}\left[n(\mu-\alpha)+\left(n \mu \alpha-\operatorname{tr} A^{2}\right)\langle x, \xi\rangle\right]$. This implies $\langle x, \xi\rangle=\mathrm{const}$ or $n \alpha^{2}=\operatorname{tr} A^{2}$. Let $\langle x, \xi\rangle=$ const. Then $\operatorname{tr}(\Delta \tilde{x})=-2 n \alpha\langle x, \xi\rangle-2 n=$ const , $\operatorname{tr}\left(\Delta^{2} \tilde{x}\right)=0$ so that the trace of $(18)$ gives $-\left(\lambda_{1}+\lambda_{2}\right) \operatorname{tr}(\Delta \tilde{x})+\lambda_{1} \lambda_{2}\left(\operatorname{tr} \tilde{x}-\operatorname{tr} \tilde{x}_{0}\right)=$ 0 . This implies $\lambda_{1} \lambda_{2}=0$ or $\operatorname{tr} \tilde{x}=\langle x, x\rangle=$ const. The latter case means $M$ is spherical, thus of 1-type, so let $\lambda_{1}=0$. Then (18) becomes $\Delta^{2} \tilde{x}=\lambda_{2} \Delta \tilde{x}$, which is impossible by (2) and (17). Now assume that $n \alpha^{2}=\operatorname{tr} A^{2}$. Then the submanifold is (a piece of) a hypersphere because $n \operatorname{tr} A^{2}=(\operatorname{tr} A)^{2}$ $+\sum_{i<j}\left(\mu_{i}-\mu_{j}\right)^{2}$ holds for any hypersurface. Moreover, the result of $[\mathrm{T}]$ and Theorem 3 imply that the quadric representation $\tilde{x}$ is 2-type if and only if hypersphere is not centered at the origin.

Remark. In [D] the author studied spherical hypersurfaces whose quadric representation is low type. Certain classification results are obtained for such hypersurfaces with $\tilde{x}$ of 2- or 3-type. For example, minimal Cartan hypersurface $\mathrm{SO}(3) / Z_{2} \times Z_{2}$ is shown to have quadric representation of 3-type i.e. this hypersurface of $S^{4}$ is of 1-type in $E^{5}$, but of 3-type in $\operatorname{SM}(5)=E^{15}$ via $\tilde{x}$.

\section{ACKNOWLEDGMENT}

This paper is essentially one chapter of the author's thesis done at Michigan State University. In that regard the author thanks Professor B. Y. Chen for continuous guidance and encouragement.

\section{REFERENCES}

[C1] B. Y. Chen, Geometry of submanifolds, Marcel Dekker, New York, 1973.

[C2] _ Total mean curvature and submanifolds of finite type, World Scientific, Singapore, 1984.

[D] I. Dimitrić, Quadric representation and submanifolds of finite type, Thesis, Michigan State Univ., 1989.

[R] A. Ros, Eigenvalue inequalities for minimal submanifolds and P-manifolds, Math. Z. 187 (1984), 393-404.

[S] K. Sakamoto, Planar geodesic immersions, Tohoku Math. J. 291 (1977), 25-56.

[T] T. Takahashi, Minimal immersions of Riemannian manifolds, J. Math. Soc. Japan 18 (1966), 380-385. 48824

Department of Mathematics, Michigan State University, East Lansing, Michigan

Current address: Department of Mathematics, Pennsylvania State University, Fayette Campus, Uniontown, Pennsylvania 15401 